\title{
Design and fabrication of ultra-wideband power amplifier based on GaN HEMT
}

\author{
Zhiqun Cheng $^{1 \mathrm{a})}$, Dandan Zhu ${ }^{1}$, Guoguo Yan ${ }^{1}$, Shuai Chen ${ }^{1}$, \\ Kai Wang ${ }^{1}$, Kaikai Fan ${ }^{1}$, Guohua Liu ${ }^{1 b)}$, \\ Hui Wang ${ }^{1}$, and Steven Gao ${ }^{2}$ \\ ${ }^{1}$ Key Lab. of RF Circuit and System, Education Ministry, \\ Hangzhou Dianzi University, Hangzhou 310018, China \\ ${ }^{2}$ School of Engineering and Digital Arts, University of Kent, UK \\ a)zhiqun@hdu.edu.cn \\ b)ghliu@hdu.edu.cn
}

Abstract: The research of an ultra-broadband power amplifier based on TGF2023-2-02 GaN HEMT which operates in the frequency ranging from $3 \mathrm{GHz}$ to $8 \mathrm{GHz}$, is presented in this paper. The transistor of GaN HEMT is modeled and a frequency compensation and multi-side impedance matching approach are adopted for broadband impedance matching of amplifier. And a fan shaped micro strip line is implemented in the input matching network to achieve the wideband higher gain features. The measured results show that the amplifier module provided more than $37 \mathrm{dBm}$ output power with minimum small signal gain of $9.8 \mathrm{~dB}$ over $3-8 \mathrm{GHz}$. The saturated output power is $38.3 \mathrm{dBm}$ under $\mathrm{DC}$ bias of $\mathrm{Vds}=28 \mathrm{~V}, \mathrm{Vgs}=-2.75 \mathrm{~V}$ at the frequency of $5 \mathrm{GHz}$.

Keywords: GaN HEMT, ultra broadband, power amplifier, output power Classification: Microwave and millimeter wave devices, circuits, and systems

\section{References}

[1] J. Lai, J. Luo and L. You: Semicond. Technol. 40 [1] (2015) 44. DOI:10.13290/ j.cnki.bdtjs.2015.01.009

[2] C. Campbell, C. Lee and V. Williams: IEEE J. Solid-State Circuits 44 (2009) 2640. DOI:10.1109/JSSC.2009.2026824

[3] G. Mouginot, H. Blanck and M. Le Pipe: IEEE IMS (2010) 1392.

[4] C. Berrached, D. Bouw and M. Camiade: The 43rd European Microwave Conference (2013) 1395.

[5] T. Wei: Internet of things technologies [5] (2012) 61.

[6] X. Xie, S. Xiao and C. Shen: J. Microwaves 29 [4] (2013) 74.

[7] D. Liu, L. Wang and X. Chen: IEEE (2012) 256.

[8] http://www.triquint.com/prducts/p/TGF2023-2-02.

[9] C. Chen, Y. Hao and L. Yang: J. Semicond. 31 (2010) 114004. DOI:10.1088/ 1674-4926/31/11/114004

[10] F. A. Mughal, A. Kashif and N. B. Cheema: IEEE (2015) 554.

[11] S. Deng, X. Chen and D. Li: Electr. Meas. Technol. 35 [8] (2012) 35.

[12] B. M. Abdrahman, H. N. Ahmed and M. E. Gouda: IEEE (2012) 5. 
[13] Z. Cheng, M. Jia, Y. Luan and X. Lian: J. Semicond. 35 [12] (2014) 125005-1-4.

[14] X. Du, Z. Shao and C. You: IEEE (2014) 638.

[15] R. Giofrè, P. Colantonio and F. Giannini: Proc. of the 7th European Microwave Integrated Circuits Conference (2012) 425.

\section{Introduction}

The power amplifier, as the most critical module in the transmitter, directly determines the overall performance of the system. Modern electronic warfare systems, personal and wireless communication, remote sensing, radar and aero spatial applications require electronic front-end systems with very high performance in terms of bandwidth, output power, and noise [1,2]. And the GaN HEMT shows clear advantages over other materials such as $\mathrm{GaAs}$ and $\mathrm{Si}$, considering its characteristics such as wide band gap, and its potential in high output power density, especially the very high drain-to-source breakdown voltage, resulting in higher device's optimum impedance, simplifying the output transformation ratio towards 50-ohm and allowing broadband design [3]. Based on GaN devices, numerous amplifiers have been presented over a period of time by many researchers. However, the bandwidth of an amplifier whose output power is larger than $5 \mathrm{~W}$ is less than 3-8 GHz. Moreover, more balanced structures are adopted in broadband amplifiers, with which a relatively high output power is obtained [4, 5]. Besides, a distributed structure is also popular with some researchers [6]. However, consistency of the transistors in the circuit is required and power consumption of the circuit is high.

In this paper, a GaN HEMT large-signal model is developed for precisely evaluating the power characteristics and designing high performance circuit. The bonding wire connected the unpackaged chip and the peripheral circuit is modeled and simulated by HFSS (high frequency structure simulator). A frequency compensation and multi-side impedance matching approach are implemented and a fan shaped micro strip line with broadband characteristics is used in the input matching network to achieve the wideband features including higher gain and output power. The actual measurements demonstrate the availability of the design approach. Good stability, high gain and better input \& output return losses have been attained in the desired band. The proposed design offers $37-38.5 \mathrm{dBm}$ output power with minimum small signal gain of $9.8 \mathrm{~dB}$ and more than $34.6 \%$ power added efficiency (PAE) over $3-8 \mathrm{GHz}$ in $\mathrm{CW}$ operating mode.

\section{Modeling}

\subsection{Device modeling}

Requirement of the accurate GaN HEMT large-signal model should account for the main effects in the device like the trapping and self-heating effects [7]. The TriQuint Materka model in Agilent ADS 2011, as the basic physical model, is adopted towards the goal of evaluating optimal device characteristics in frequency range of $1-10 \mathrm{GHz}$ in this paper. The model is based on the equivalent circuit topology shown in Fig. 1, including two sections: extrinsic and intrinsic elements. The small signal parameters of the model are extracted at first based on measured 
S-Parameter all of frequency range and linear model parameters for $1.25 \mathrm{~mm}$ unit GaN cell given by the datasheet [8]. The large-signal model can be achieved based on small signal model and measured output impedances of several frequencies under large signal. There are several intrinsic capacitances such as gate-to-source Cgs, gate-to-drain Cgd and drain-to-source Cds and resistor Rin changed to large signal behavior, which are extracted through comparison of measured versus simulated saturated power (Psat), PAE and the optimal load impedance (Ropt) under load-pull operation. The remaining parameters' values of such model are also needed to be optimized for predicting accurately device characteristics such as threshold voltage, coefficient for pinch-off change with respect to Vds and saturation drain current, etc. Table I exhibits the corresponding extrinsic and intrinsic elements' values finally obtained under small signal and large signal operation respectively.

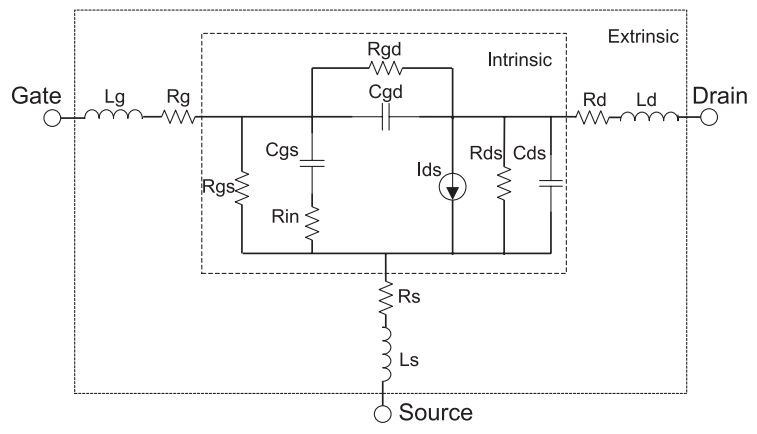

Fig. 1. Equivalent-circuit model topology used.

Table I. Extrinsic and intrinsic elements' values of the model

\begin{tabular}{|l|c|c|}
\hline Element & Small-signal model & Large-signal model \\
\hline Cgs (pF) & 1.79 & 7.28 \\
\hline Cds (pF) & 0.402 & 0.576 \\
\hline Cgd (pF) & 0.064 & 0.209 \\
\hline Rin (ohm) & 0.26 & 0.06 \\
\hline Rg (ohm) & \multicolumn{2}{|c|}{0.786} \\
\hline Rd (ohm) & \multicolumn{2}{|c|}{0.15} \\
\hline Rs (ohm) & \multicolumn{2}{|c|}{0.0089} \\
\hline Lg (nH) & \multicolumn{2}{|c|}{0.028} \\
\hline Ld (nH) & \multicolumn{2}{|c|}{0.012} \\
\hline Ls (nH) & \multicolumn{2}{|c|}{19750} \\
\hline Rgs (ohm) & \multicolumn{2}{|c|}{13.05} \\
\hline Rgd (ohm) & \multicolumn{2}{|c|}{} \\
\hline Rds (ohm) & \multicolumn{2}{|c|}{} \\
\hline
\end{tabular}

The validation of such model is performed through comparison of measured versus simulated S parameters and power characteristics. Fig. 2 shows the fitting of $\mathrm{S}$ parameters. Table II shows the comparison of Psat, PAE and Ropt at 3 and $10 \mathrm{GHz}$, and the measured results are given by datasheet [8]. The simulated small signal parameters and power characteristics both have a good agreement with the measured ones. 

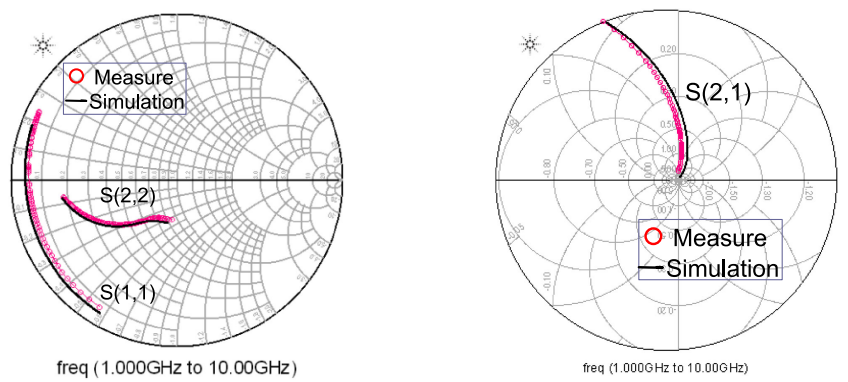

Fig. 2. Measured and modeled S-Parameter.

Table II. Measured and modeled large-signal performance.

\begin{tabular}{|c|c|c|c|c|c|c|}
\hline & \multicolumn{4}{|c|}{$3 \mathrm{GHz}$} & \multicolumn{3}{c|}{$10 \mathrm{GHz}$} \\
\cline { 2 - 7 } & $\begin{array}{c}\text { Psat } \\
(\mathrm{dBm})\end{array}$ & $\begin{array}{c}\text { PAE } \\
(\%)\end{array}$ & $\begin{array}{c}\text { Ropt } \\
(\mathrm{ohm})\end{array}$ & $\begin{array}{c}\text { Psat } \\
(\mathrm{dBm})\end{array}$ & $\begin{array}{c}\text { PAE } \\
(\%)\end{array}$ & $\begin{array}{c}\text { Ropt } \\
(\mathrm{ohm})\end{array}$ \\
\hline Simulated & 41.39 & 52.77 & $18.788-\mathrm{j} * 14.724$ & 38.9 & 50.6 & $9.294-\mathrm{j} * 1.653$ \\
\hline Measured & 40.1 & 60.3 & $20.4-\mathrm{j} * 15.5$ & 39.5 & 57.8 & $10.3-\mathrm{j} * 2.5$ \\
\hline
\end{tabular}

\subsection{Model of bonding wire}

The bonding wire is modeled and simulated by HFSS so as to accurately estimate the effect of the bonding wire for the circuit. Fig. 3(a) is the model of the bonding wire in HFSS. Pad geometry and material properties of transistor and PCB substrates are taking to the account in the wire bond simulations. No. 1 and No. 3 respectively represent ports of connecting bonding wires and the micro strips. No. 2 and No. 4 respectively represent ports of connecting bonding wires and the chip pads. The diameter of the bonding wire of gold is $25 \mathrm{um}$, the span is $400 \mathrm{um}$, the height is $100 \mathrm{um}$ and the distance between is $260 \mathrm{um}$. Bonding wire model is given out in the simulation software of ADS. But, there are big errors in the broadband frequency range. Fig. 3(b) compares the simulated reflection coefficient $\mathrm{S}(1,1)$ of port 1 in HFSS and ADS in the frequency from 3-8 GHz. So, simulation results of HFSS are used for broadband amplifier design.

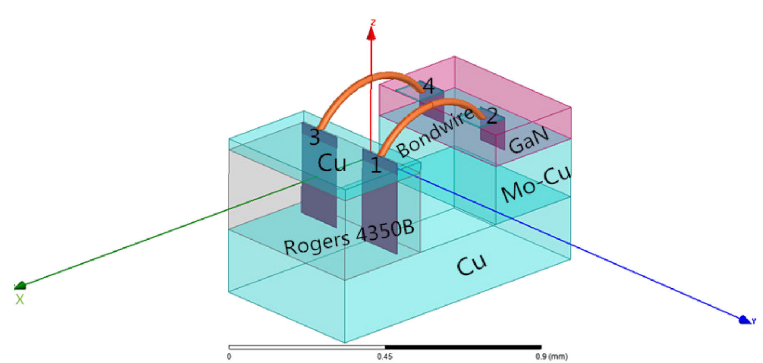

(a)

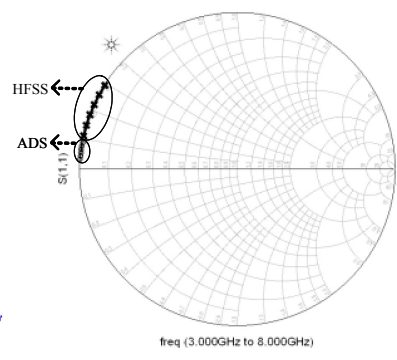

(b)

Fig. 3. EM analysis of bonding wire: (a) model; (b) simulated.

\section{Broadband power amplifier design}

The wideband amplifier is designed in the software ADS. And its schematic diagram is shown in Fig. 4. The circuit consists of the matching circuits, DCbiased circuit and GaN HEMT. The DC bias and matching networks are all on the 
printed circuit board (PCB), which is a Rogers $4350 \mathrm{~B}$ with a higher dielectric constant of 3.66 which benefits for the small size of the matching components and a substrate thickness of $0.254 \mathrm{~mm}$. Moreover, it is necessary to leave an appropriate hole for the chip to ensure the chip surface and micro strip line in the same plane. Both the input and output ports are connected to the standard $50 \Omega$ load.

The output matching network of power amplifier is designed according to optimal impedance of the load-pull based on the model of the RF power transistor to guarantee optimal performance under large signal operation. Based on these values, shown in Fig. 5, multi-side transmission line elements with different characteristic impedance, whose continuous changes are conductive to the realization of broadband matching, are optimized for the optimal load matching at transistor output to obtain a flat behavior in the bandwidth. This structure benefits well for the small size of the layout compared with the only use of multi stepped impedance transformer which employs quarter wave transmission line, as seen in [1]. In addition, the fan shaped Stub2 is opened for the odd harmonic waves and closed to the even harmonic waves, which is useful to prevent the leakage of energy from the bias network and promote the linearity and output power of the amplifier. It can also improve the microwave performance of the power device in output power and gain [9].

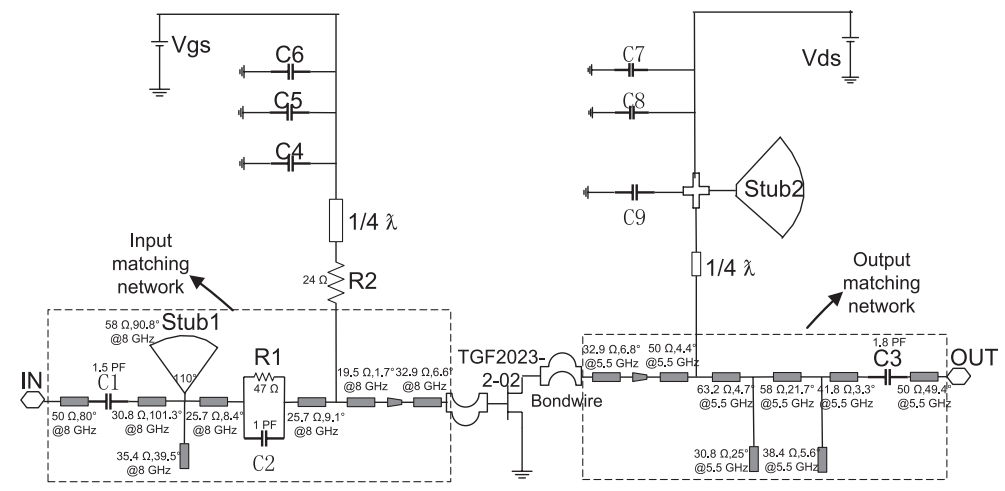

Fig. 4. Schematic diagram of the proposed power amplifier.

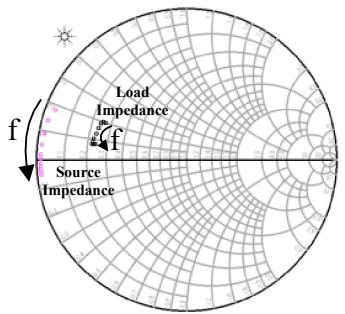

Fig. 5. Broadband load and source impedance trajectories evaluated in the frequency range 3-8 GHz.

An important effort for input matching was required to control the gain flatness over 3 to $8 \mathrm{GHz}$. R1 and R2 are used to achieve required stability within the band of interest. But improved stability and better VSWR are achieved at a cost of gain. Therefore, to compensate the gain, a parallel capacitor $\mathrm{C} 2$ is used along with the series resistor R1. This arrangement is called hybrid stability network which makes it possible to attain stability and broadband matching simultaneously for R1 and C2 respectively tuning the gain of low and high frequency signal in the operating band 
[10]. The resistor R2 between DC power supply and the transistor gate for the GaN HEMT neither consumes any power nor produces any heat, because the gate current is nearly zero under ideal conditions. The input and output coupling capacitors are shown as $\mathrm{C} 1$ and $\mathrm{C} 3$ respectively, which can insulate the DC current and match simultaneously.

A frequency compensation approach for the optimum matching at frequency of $8 \mathrm{GHz}$ is applied. A fan shaped Stub1 with the characteristic of broadband impedance transformer is used during the multi-side impedance matching design. One of the important features of the fan shaped stub is that its input port can be in accordance with the needs of any small, which makes the contact point easily defined. Besides, compared with the equivalent general stub, its reactance, which is presented in [11], varies slowly with frequency near the resonance point, resulting in a wider bandwidth. The validity of the method is demonstrated by the example shown in Fig. 6, which has a comparison of a 90 degrees radial stub and a traditional direct form of stub with the quarter wave length at $5.5 \mathrm{GHz}$. Both the two stubs, whose simulation structures and parameters can be attained through theoretical calculation, have the same input impedance, as shown in Fig. 6(a). Fig. 6(b) shows respectively the comparison results of the reflection coefficient $S$ $(1,1)$ and the transmission coefficient $S(2,1)$ of the two stubs, which both exhibit better performance of the fan shaped stub including more smooth $\mathrm{S}(1,1)$ curve and lower transmission coefficient. In addition, the physical length of the sector stub is only about $1 / 2$ of the equivalent straight stub, leading to a smaller area in substrate. Furthermore, compared with general multi-side impedance matching, the use of sector structure can obtain equal RF performance with less parallel stubs, which greatly reduces the size of the layout. This comparison can be seen in [12].
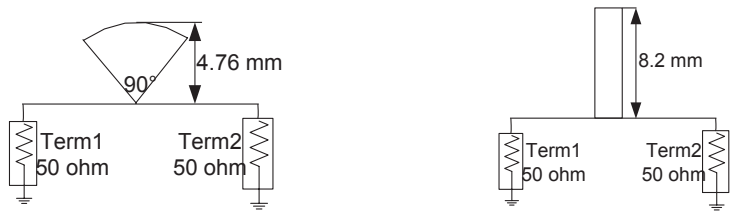

(a)
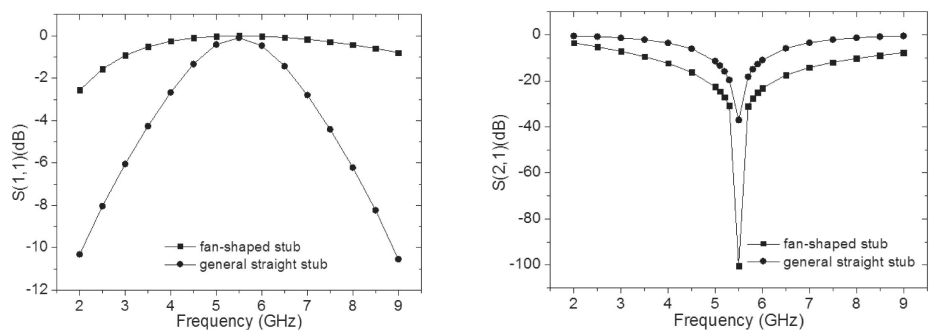

(b)

Fig. 6. Comparison of the fan-shaped stub and general straight stub performance: (a) simulated structures; (b) reflection coefficient $\mathrm{S}(1,1)$ and the transmission coefficient $\mathrm{S}(2,1)$.

\section{Results and discussion}

Fig. 7 shows photos of the power amplifier module. Resistors and capacitors with SMD are mounted on the PCB by silver paste. The bonding wires are used to 
connect the GaN HEMT with the micro strip on the PCB, and a Mo-Cu plate which thermal expansion coefficient is between SiC (substrate of GaN HEMT) and metal copper (as a heat sink) is used as the chip's carrier. What's more, because the GaN HEMT is a depleted field effect transistor, it must be supplied by double power supplies. And a negative voltage of the gate must be turned on at first and then a positive voltage of the drain is turned on. The negative voltage switches off after positive voltage. This can ensure the transistor to work safely. The power amplifier is measured under DC bias of $\mathrm{Vds}=28 \mathrm{~V}, \mathrm{Vgs}=-2.75 \mathrm{~V}$.

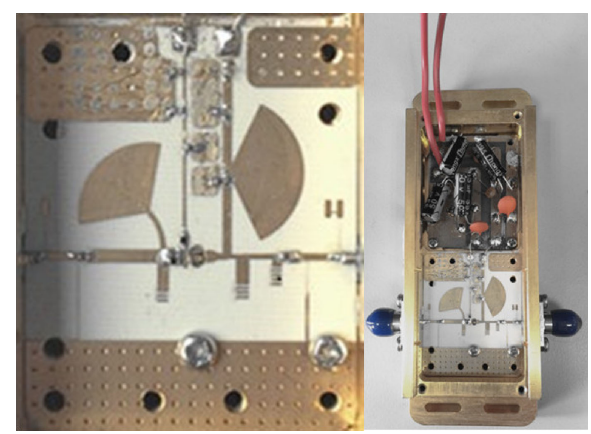

Fig. 7. Image of the fabricated wideband amplifier.

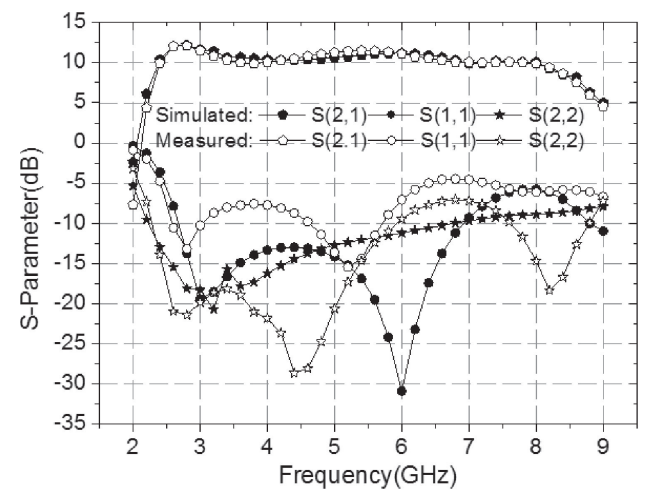

Fig. 8. Simulated and measured S-parameter of the circuit.

Agilent E4447A vector network analyzer is used to measure small signal S parameters. Fig. 8 shows the measurement and simulation S-parameters of the proposed amplifier. The measurement results show that $\mathrm{S}(2,1)$ is more than $9.8 \mathrm{~dB}$, input return loss $\mathrm{S}(1,1)$ is less than $-4.6 \mathrm{~dB}$, output return loss $\mathrm{S}(2,2)$ is from -7.1 to $-28.7 \mathrm{~dB}$ over 3 to $8 \mathrm{GHz}$. The measured results are close to simulated results. They have the same change trend in the all of band and show good spread behaviour. And it has an improvement than before [13]. Agilent E8257C signal generator, Agilent E4416A power meter and Mini driving amplifier are used to measure large signal power. Fig. 9 shows the measured and simulated $\mathrm{CW}$ output power, power gain and PAE at the frequency of $5 \mathrm{GHz}$. It shows that the measured maximum output power of $38.3 \mathrm{dBm}$ with the corresponding power gain of $9 \mathrm{~dB}$. The measured results of the saturated output power, power gain, and PAE versus frequency are drawn in Fig. 10. It can be seen that the proposed design offers $37-38.5 \mathrm{dBm}$ output power and more than $34.6 \%$ PAE with a maximum of $49.3 \%$ over 3-8 GHz. Table III gives a list of the measured results from reported literature 
and our work. This amplifier has a better performances including broadband and out power than others.

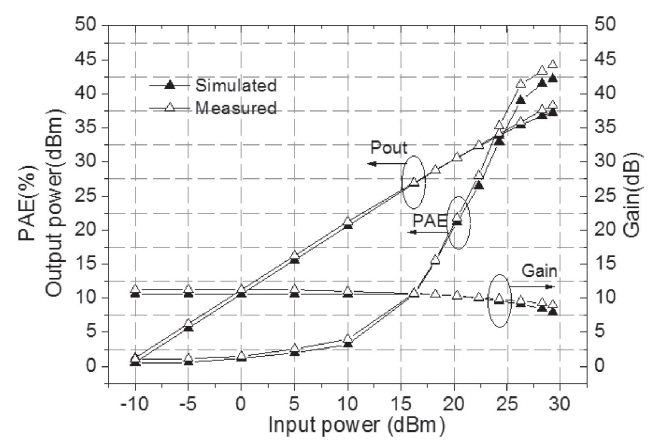

Fig. 9. Output power, gain and PAE versus input power of the circuit.

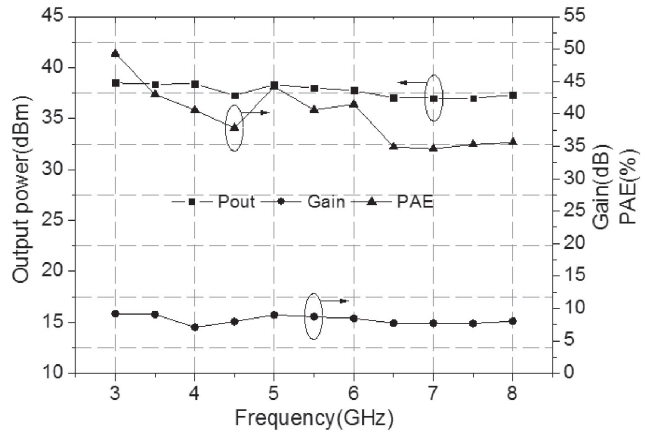

Fig. 10. Saturated output power, power gain and PAE versus frequency.

Table III. Comparison of characteristics of amplifiers

\begin{tabular}{|l|c|c|c|l|}
\hline Ref. & Frequency $(\mathrm{GHz})$ & output power $(\mathrm{dBm})$ & PAE $(\%)$ & Transistor \\
\hline$[4]$ & $1-3$ & 45.8 & $36-44$ & GaN HEMT \\
\hline$[6]$ & $0.3-2.5$ & 39 & $15-35$ & GaN HEMT \\
\hline$[12]$ & $0.8-4.2$ & 40 & 28 & GaN HEMT \\
\hline$[14]$ & $1-3$ & $37.7-41.7$ & $54.3-71.4$ & GaN HEMT \\
\hline$[15]$ & $1-7$ & 36 & 33 & GaN HEMT \\
\hline This work & $3-8$ & $37-38.5$ & $34.6-49.3$ & GaN HEMT \\
\hline
\end{tabular}

\section{Conclusion}

A wideband amplifier is designed using a novel method of wideband matching networks at frequency ranging over 3 to $8 \mathrm{GHz}$. The amplifier is tested for the small signal and large signal characteristics. The results show that the proposal amplifier has the good performances at the broadband frequency range. The very good agreement between simulation results and measured data shows the accuracy of the model and also the efficiency of the design approach. All these performance are promising for the future development on ultra-wideband power amplifier.

\section{Acknowledgments}

This material is based upon work funded by Zhejiang Provincial Natural Science Foundation of China under Grant No. LZ16F010001. 